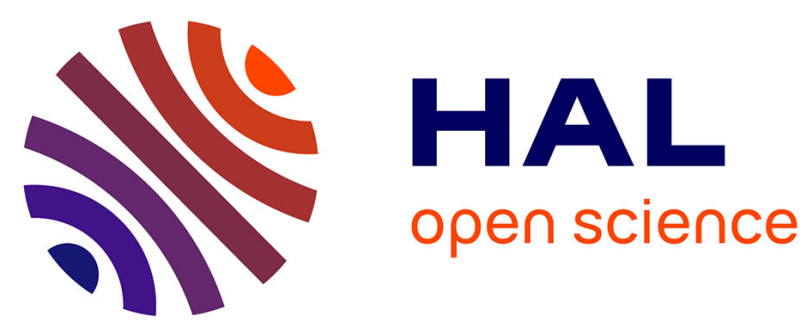

\title{
Transmission of HIV-1 minority-resistant variants and response to first-line antiretroviral therapy.
}

Olivia Peuchant, Rodolphe Thiébaut, Sophie Capdepont, Valerie

Lavignolle-Aurillac, Didier Neau, Philippe Morlat, François Dabis, Hervé

Fleury, Bernard Masquelier

\section{To cite this version:}

Olivia Peuchant, Rodolphe Thiébaut, Sophie Capdepont, Valerie Lavignolle-Aurillac, Didier Neau, et al.. Transmission of HIV-1 minority-resistant variants and response to first-line antiretroviral therapy.: Transmission of minority resistant HIV-1. AIDS. Official journal of the international AIDS Society, 2008, 22 (12), pp.1417-23. 10.1097/QAD.0b013e3283034953 . inserm-00270200

\section{HAL Id: inserm-00270200 https://www.hal.inserm.fr/inserm-00270200}

Submitted on 4 Apr 2008

HAL is a multi-disciplinary open access archive for the deposit and dissemination of scientific research documents, whether they are published or not. The documents may come from teaching and research institutions in France or abroad, or from public or private research centers.
L'archive ouverte pluridisciplinaire HAL, est destinée au dépôt et à la diffusion de documents scientifiques de niveau recherche, publiés ou non, émanant des établissements d'enseignement et de recherche français ou étrangers, des laboratoires publics ou privés. 
Transmission of HIV-1 minority resistant variants and response to first-line antiretroviral therapy

O Peuchant ${ }^{1}$, R Thiébaut ${ }^{2}$, S Capdepont ${ }^{1}$, V Lavignolle-Aurillac ${ }^{2}$, D Neau ${ }^{2,3}$, P Morlat ${ }^{3}$, F Dabis ${ }^{2}$, H Fleury $^{1}$, B Masquelier ${ }^{1}$ and the ANRS CO3 Aquitaine Cohort *

1 CHU de Bordeaux, Laboratoire de Virologie, and Université Victor Segalen Bordeaux 2, EA 2968, F-33076, Bordeaux, France

2 INSERM U593 and U875, ISPED, Université Victor Segalen, F-33076, Bordeaux, France

3 CHU de Bordeaux, Département de Maladies infectieuses, Bordeaux, France

* Participants listed in appendix

Short title: Transmission of minority resistant HIV-1

1 Table, 2 Figures

Text : 3483 words

Abstract : 250 words

Presented in part at the $14^{\text {th }}$ Conference on Retroviruses and Opportunistic infections, Los Angeles , CA, USA, February 25-28, 2007. Abstract 666. 


\section{Abstract}

Background: The transmission of drug-resistant HIV-1 can impair the virological response to antiretroviral therapy (ART). Minority resistant variants (RV) have been detected in acute seroconverters. We investigated the clinical relevance of the detection of majority and of minority RV in an observational study in ART- naïve, recently infected patients.

Methods: We included patients infected between 1996 to 2005, with a plasma sample obtained $<18$ months after seroconversion and prior to ART initiation. Majority RV were determined by direct population sequencing. Minority RV were searched by allele-specific PCR for the mutations K103N and M184V in RT and L90M in protease. The association between resistance and viro-immunological response to ART was estimated by using a piecewise linear mixed model.

Results: Majority RV were detected in 23/172 (13.4\%) patients. Patients with majority RV had a lower mean plasma viral load (VL) and higher mean CD4 cell count at baseline compared to those without resistance. The decrease in VL between one and 6 months on ART was significantly steeper in patients with sensitive viruses compared to patients with majority RV ( $\mathrm{p}=0.029)$. Minority RV were detected in 21/73 (29\%) patients with wild-type viruses at sequencing analysis. The presence of minority RV did not modify baseline VL and CD4 cell count, and did not affect the changes in VL and CD4 cell count.

Conclusion: The transmission of majority RV, but not of minority RV, influenced the response to ART in this prospective study. The detection of the transmission of minority RV warrants further clinical validation.

Key words: HIV-1 drug resistance, transmission, antiretroviral therapy, minority variants 


\section{Introduction}

The selection of HIV-1 variants with resistance to antiretroviral drugs (ARV) is one important factor limiting the effect of antiretroviral therapy (ART). In ART-treated patients with detectable plasma HIV-1 RNA, the prevalence of resistance to at least one drug can reach up to $80 \%[1,2]$. The transmission of drug resistant HIV-1 has been widely documented and the prevalence of resistance in drug -naïve patients has been shown to reach $10 \%$ or more in Western Europe and in North America [3-5]. Phylogenetic analyses have shown that transmitted resistant variants could originate not only from treated patients but also from acutely-infected, drug-naïve patients [6]. Some studies have shown the impact of the transmission of resistant variants leading to worse virological and immunological responses to first-line ART [7-9], but other studies failed to show such an influence [10].

The detection of transmitted resistant variants has been processed in the vast majority of studies by using conventional nucleotide sequencing methods after PCR amplification of the viral genome from plasma RNA. However, these bulk sequencing procedures cannot detect minority variants below a detection threshold of $20 \%$ of the global population. The detection of minor populations of drug-resistant HIV-1 has been recently reported in acute seroconverters [11]. Thus even in the absence of resistance in the current dominant genotype, the possibility remains that minority species may exist against which the treatment will prove less effective.

In this study we report the detection of majority resistant variants and of minority resistant variants in a cohort of recently HIV-1 infected patients, and we study the influence of majority and of minority resistant variants on subsequent response to first-line ART. 


\section{Patients and Methods}

\section{Study population}

The patients included in this retrospective study were HIV-1 infected patients followed-up at the Bordeaux University Hospital within the ANRS CO 3 Aquitaine Cohort. The Aquitaine Cohort is a prospective hospital-based cohort of HIV-1-infected patients under routine clinical management [12], initiated in 1987 in the Bordeaux University Hospital and four other public hospitals in Aquitaine by the Groupe d'Epidémiologie Clinique du Sida en Aquitaine (GECSA). Inclusion criteria were: all adults who were in- or out-patients of the participating hospital wards with HIV-1 infection confirmed by Western blot testing, regardless of clinical stage, either having at least one follow-up after the first report or with a known date of death, and having given informed consent. Patients were included in the present study if they had a reliably estimated date of HIV-1 seroconversion between 1996 and 2005, either with a laboratory evidence of acute infection (antibody negative with plasma HIV-1 RNA and/or p24 antigen positivity, or evolving antibody response), or with previously seronegative test within 3 years of the first seropositive test. In this latter case, the estimated date of seroconversion was the mid point between the last negative and the first positive serology. Included patients had plasma HIV-1 RNA and CD4 cell count measurements available before therapy and at least at one time point during the first six months on ART.

Among the patients with an accurate estimation of their date of HIV infection between 1996 and 2005 and starting ART, conventional genotypic resistance analysis could be processed in a subgroup of patients with available sample before ART and viral load and CD4 cell counts measurements on ART. The study of minority resistant variants could then be processed on a subgroup of patients originating from this initial study and with available sample and no genotypic resistance detected by sequence analysis.

\section{Genotypic Resistance Analysis}


Genotypic resistance analysis was processed on the $1^{\text {st }}$ available plasma sample within 18 months of the estimated date of seroconversion and before initiation of ART. HIV-1 reverse transcriptase (RT) and protease genes were sequenced according to the Agence Nationale de Recherche sur le SIDA (ANRS) consensus protocol; the details of the methods appear at www.hivfrenchresistance.org. Resistance mutations were reported as listed by the International AIDS Society-USA Panel (www.iasusa.org, update October 2005), including revertant mutations at RT codon 215, and/or the ANRS algorithm version 2006 (www.hivfrenchresistance.org), excluding minor protease mutations and the V118I RT mutation. Genotypic resistance analysis was processed retrospectively for most patients, because these tests are recommended in France for all antiretroviral -naïve patients only since 2006[13] (ref Yeni). Genotypic resistance analysis results were available before the choice of regimen only for patients with diagnosis of HIV-1 primary infection since 2000.

\section{Quantification of minority resistant variants by allele-specific PCR}

Minority populations of drug-resistant variants were detected from plasma samples by realtime, allele-specific PCR (ASPCR) for the RT mutations K103N and M184V and the protease mutation L90M. ASPCR was processed on a subgroup of patients originating from the initial group with conventional genotypic resistance analysis, with available plasma samples and/or RT-PCR products and no resistance to the subsequent ART regimen as detected by direct sequencing. We used a method adapted from Metzner et al [11]. RT and protease were first reverse transcribed and PCR-amplified from plasma RNA, as described above for the genotypic resistance analysis. The PCR products were then submitted to nested real-time PCR enabling the specific amplification of wild type or mutated sequences, or whole population for each considered mutation. For the detection of the K103N mutation, the primers were IN K103N 5'-CCGCAGGGTtAAAAAAGAIC-3' (nt 2839-2858) and Pol 3002 5'CTGTGGAAGCACATTGTACTG-3'(nt 2982-3002) for detection of the mutated population, 
and Pol 2801 5'-TCAAGACTTCTGGGAAGTTCA-3' (nt 2801-2821) and Pol 3122 5'TGCTGCCCTATTTCTAAGTCA-3' (nt 3122-3134) for the amplification of a 334 bp fragment corresponding to the whole viral population and comprising RT codons 103 and 184. For the detection of the mutation M184V, the primers IN M184V 5'CCAGACATAGTTATCTATCAATAIG-3' (nt 3075-3099) and N35 5’CCTACTAACTTCTGTATGTCATTGACAGTCCAGCT-3’ (nt 3300-3333) enabled the detection of the mutated population, and Pol 2801 and Pol 3122 the detection of the whole population. For the detection of the L90M protease mutation, the primers were Pol 2316 5'-GCTCTATTAGATACAGGAGCAG-3' (nt 2316-2337) and either INL90M 5'TGCAACCAATCTGAGTCIT-3’ (nt 2520-2538) for the detection of the mutated population, or INL90 5'-TGCAACCAATCTGAGTCIA-3'for the detection of the wild type population. In order to overcome the possibility of non-amplification due to subtype-specific or unspecific polymorphic variations in the HIV genome, when we designed primers screened by ASPCR we took into account the polymorphic variations observed in the bulk sequencing results. The real-time PCR reactions were performed on the Light Cycler 1.5 with the LightCycler FastStart DNA Master SYBR Green I ${ }^{\circledR}$ kit (Roche Diagnostics, Meylan, France). For the quantitation of the different viral populations, DNA standards were prepared by PCR amplification of the RT or protease gene of different pNL4-3 HIV-1 plasmids carrying or not the mutations L90M, K103N and M184V (gift from Dr François Clavel, INSERM U552, Paris, France). The presence or absence of each mutation in the corresponding PCR products was confirmed by sequencing. The standards were tested in duplicate for each experiment, and the standards were prepared by serial dilution from $10^{6}$ to $10^{2}$ copies per reaction. The viral PCR products were tested in triplicate at three different dilutions $\left(10^{-1}, 10^{-2}\right.$ and $\left.10^{-3}\right)$. The specificity of the detection of the amplified product was checked using the analysis of the melting curve for each reaction. The ASPCR result was expressed as the percentage of 
mutated sequences reported to the whole (mutated + wild type) sequences \pm standard deviation. The discriminatory ability of each assay was tested as previously described [14], in reciprocal experiments by adding $10^{6}$ copies of non complementary DNA (10 -10 copies) of either wild type or mutant DNA with the oligonucleotides specific to the wild type or mutated (for L90M) or to the whole population and mutated (for M184V and K103N) population, taken into account the standard deviation.

Samples presenting mutations detected by bulk sequencing analysis and samples scored as wild type by cloning analysis were used as positive and negative controls.

The minority resistant study was done all retrospectively, and could thus not influence the choice of treatment regimens.

\section{Evaluation of the ASPCR assay}

The detection of minority resistant variants within the global plasma viral population was assessed by using the ASPCR. This method uses allele-specific oligonucleotides with discriminatory abilities for the detection of either wild-type or mutant sequences. In order to overcome non-specific amplification with wild-type primers at RT codons 103 and $184 \mathrm{~V}$, we modified the technique published by Metzner et al [11] by quantifying the global viral population instead of the wild type population. The sensitivities of the assay for detecting a mutated sequence out of the global population were shown to be $0.08 \%, 0.4 \%$ and $0.3 \%$ for the mutations L90M, K103N and M184V, respectively. The specificity and sensitivity of the method were always successfully checked on positive and negative controls.

\section{Quantification of plasma HIV-RNA}

Plasma HIV-1 RNA was measured using bDNA (Quantiplex ${ }^{\circledR}$, Bayer Diagnostics, Eragny, France, threshold 500 or 50 copies/ml) or RT-PCR (Cobas TaqMan ${ }^{\circledR}$, Roche Diagnostics, Meylan, France, threshold 40 copies/ml). 


\section{Statistical methods}

The association between genotypic resistance and plasma HIV-1 RNA or CD4 levels was estimated at treatment initiation and during the first six months on ART. Repeated measurements of HIV RNA and CD4+ cell count were analysed using a piecewise linear mixed model accounting for the intra-patient correlation. Resistance was defined as genotypic resistance to at least one drug of the ART regimen. The effect of resistance was tested on the baseline value at ART initiation, on the short-term slope (until one month) and on the longterm slope (after one month). Furthermore, the estimation of model parameters took into account the left-censoring of HIV RNA due to undetectable measures [15]. Models were adjusted for the type of assay used to quantify plasma HIV RNA. All analyses were performed with SAS Software (SAS v9.0, SAS Institute, Cary, NC, USA). We studied separately the influence of majority resistant variants and of minority resistant variants.

\section{Results}

\section{Patients' characteristics and drug resistance in the bulk viral population}

Among 295 patients with an accurate estimation of their date of HIV infection between 1996 and 2005 and starting ART, 172 patients had an available genotype before ART and viral load and CD4 cell counts measurements on ART and were therefore included in the study. The characteristics of the included patients are shown in table 1 and were not different from those of the initial population. The median time from estimated seroconversion to the date of sampling for resistance genotypic analysis was 5.2 months (IQR: 1.7-10.4). Genotypic resistance was detected by sequence analysis in 23/172 (13.4\%) HIV-1 strains. Resistance to NRTIs was detected in 20 patients, resistance to NNRTIs in 11 patients and resistance to PIs in seven patients. Fifty patients were treated by 2-3 NRTIs, 83 patients received PI-containing ART, and 39 received NNRTI-based ART. Twenty-two (12.8\%) patients received less than three ARVs. The characteristics of patients, as shown in table 1, did not significantly differ 
according to the presence or absence of genotypic resistance. The median delay between the date of seroconversion and the date of beginning ART was 9.7 months (IQR 2-16) and 11.9 months (IQR: 5-15) in patients with sensitive and in patients with resistant viruses, respectively ( $p=0.41)$. Finally, $16(9.3 \%)$ patients were infected by an HIV-1 strain presenting genotypic resistance to at least one drug comprised in the subsequent ART regimen.

\section{Transmission of minority resistant variants}

The research of minority resistant variants could be processed for 73 patients originating from the 172 patients with conventional genotypic resistance analysis, who presented similar characteristics to the whole population and were treated by: 2-3 NRTIs ( $n=21$ ); two NRTIs + one NNRTI $(n=14)$, or two NRTIs + one PI $(n=38)$. Ten of $73(13.7 \%)$ patients were infected by HIV-1 non-B subtypes. Mutations were searched by ASPCR when they were not detected by direct sequencing and had a putative impact on response to ART. Minority variants were detected in 21/73 (29\%) patients: K103N was detected in 3/14 (21\%) patients, M184V in 18/72 (25\%) and L90M in 0/40 (0\%) patients. Minority variants represented $2.5 \%$ to $7.1 \%$ of the total viral population for the mutation $\mathrm{K} 103 \mathrm{~N}$ and $1.3 \%$ to $23.5 \%$ for $\mathrm{M} 184 \mathrm{~V}$. In patients for whom the M184V was detected as minority population, the subsequent ART regimens were non boosted-PI-based $(n=5)$, boosted-PI-based $(n=5)$, NRTI-based $(n=5)$, and efavirenz-based $(n=3)$.

\section{Association between transmitted resistance and response to ART}

\section{Majority variants and response to ART}

The association between the transmission of majority resistant variants detected by direct sequencing and the virological and immunological outcome on ART was studied on 172 patients with a 6-month follow-up on ART. The median number of viral load and CD4 measurements in the first 6 months was three. The influence of majority resistant variants on 
virological response is shown in Figure 1A. At baseline before ART, the baseline plasma HIV-1 RNA was significantly lower in the resistant group compared to the susceptible group (3.81 $\log _{10}$ copies/ml vs $4.68 \log \log _{10}$ copies/ml, $\mathrm{p}=0.002$ ). There was then no difference in the decay of plasma HIV-1 RNA between baseline and month $1\left(-1.93 \log _{10}\right.$ copies/ml for resistant group vs $-2.04 \log _{10}$ copies $/ \mathrm{ml}$ for susceptible group, $\mathrm{p}=0.48$ ). However, the continuing decrease in viral load was significantly steeper in the susceptible group compared to the mutated group between month 1 and $6\left(-0.43 \log _{10}\right.$ copies/ml/month vs $-0.11 \log _{10}$ copies $/ \mathrm{ml} / \mathrm{month}, \mathrm{p}=0.029)$.

The baseline CD4 $\left(^{+}\right)$cell count was significantly higher in the resistant group compared to the susceptible group (560 cells/ $\mu \mathrm{l}$ vs 438 cells $/ \mu \mathrm{l}, \mathrm{p}=0.04$ ) (Figure $1 \mathrm{~B}$ ). The two groups had then parallel increases in CD4 $(+)$ cell counts on ART leading to the same difference in CD4+ level at 6 months (682 cells/ $\mu \mathrm{l}$ vs 533 cells/ $\mu \mathrm{l}, \mathrm{p}=0.04)$.

Seven patients presented at least one of three mutations $184 \mathrm{~V}, 103 \mathrm{~N}$ or $90 \mathrm{M}$ as a majority mutation population and tended to have a lower baseline HIV RNA load (difference -0.67, $\mathrm{p}=0.08)$ and the change in HIV RNA at months 1 and 6 was similar $(\mathrm{p}=0.65$ and $\mathrm{p}=0.79)$ compared to the patients without mutations.

\section{Minority resistant variants and response to ART}

The influence of the minority resistant variants was studied in a subgroup of 73 patients. The resistant group (with minority resistant variants) and the susceptible group (with no resistance to the ART regimen) had comparable plasma HIV-1 RNA levels at baseline (4.71 $\log _{10}$ copies/ml vs $4.81 \log _{10}$ copies $/ \mathrm{ml}, \mathrm{p}=0.68$ ) (Figure $2 \mathrm{~A}$ ). There was then no difference in the evolution of the plasma HIV-1 RNA in the two groups between baseline and month 1 $\left(-1.99 \log _{10}\right.$ copies/ml in susceptible group vs $-2.17 \log _{10}$ copies/ml in resistant group, $\mathrm{p}=0.49)$ and between month 1 and month $6\left(-0.54 \log _{10}\right.$ copies/ml in susceptible group vs $0.35 \log _{10}$ copies $/ \mathrm{ml}$ in resistant group, $\mathrm{p}=0.23$ ) 
The comparison of the baseline CD4 $(+)$ cell counts in the two groups showed a non significant trend towards higher CD4 $(+)$ cell counts in the resistant group compared to the susceptible group (451 cells/ $\mu \mathrm{l}$ vs 534 cells/ $\mu \mathrm{l}, \mathrm{p}=0.14$ ) (Figure 2B). No significant difference in the evolution of the CD4 $(+)$ cell counts could was then shown between the two groups ( $\mathrm{p}=0.50$ during the first month and $\mathrm{p}=0.52$ later on).

\section{Discussion}

In our cohort of patients with recent HIV-1 infection and subsequent ART, enrolled between 1996 to 2005, we could detect the transmission of resistant HIV-1 in $13.4 \%$ of patients by using a conventional sequencing method. This prevalence is comparable to other studies in France or in Europe in the same period [3, 4, 7]. We then investigated the transmission of minority resistant variants in a subgroup of patients with no resistance detected by bulk sequencing. We used a modified version of the ASPCR method in order to detect accurately both the minority mutated genomes, with a limit of detection at $<0.5 \%$ of the total population, and the total number of amplified genomes. We detected minority mutated variants associated with resistance to the subsequent ART in $29 \%$ of patients. This finding is in accordance with previous data obtained in acute seroconverters [11] and suggests that minority resistant variants can be detected not only at the onset of the primary infection but also later on. Similar data were recently reported from an American cohort of antiretroviralnaïve patients [16] showing that low frequency mutations were equally found in recentlyinfected persons and infections of longer duration. This result is also in agreement with model predictions according to the rapid turnover, the high mutation rate and large viral population size [17]. We cannot rule out the possibility that the minority variants could have been generated de novo after transmission, even if, considering the loss of viral fitness conferred by M184V there is no reason that this mutation could have been selected in absence of drug, even at a minority level. 
It is important to note that our results were obtained by investigating only three mutations (K103N and M184V in the RT and L90M in the protease), thus the estimation of the frequency of minority resistant variants would have been certainly higher by searching for more mutations. When we studied the virological response in the group of patients having K103N, M184V or L90M as majority population, we could not show any difference in comparison with the group of patients without mutations. However, according to the restricted number of patients with the mutations of interest, the statistical power for finding a significant difference was limited (about 60\% for finding a difference of $0.5 \log 10$ copies $/ \mathrm{mL}$ at a given time).

The mutation more often detected by ASPCR in our study was the M184V mutation. We have two explanations for this finding: first, we showed previously that M184V was the most frequent mutation in patients on ART with detectable viral load [1], thus this mutation is likely to be frequently transmitted; second, the M184V mutation confers an impaired replicative capacity to the virus [18], and in absence of 3TC or FTC, the M184V variants rapidly disappear in the majority population.

We could investigate the virological and immunological responses to ART in our patients according to the presence or absence of resistant variants in the majority or only in the minority population. The detection of a baseline resistance in the majority population was shown to be associated with a less pronounced decrease in plasma HIV-1 RNA between one and six months on therapy. This finding is in accordance with previous studies, suggesting that transmitted resistance could lead to sub-optimal response to first-line therapy, supporting the guidelines which recommend performing genotypic resistance analysis prospectively in all patients at the time of diagnosis of the HIV infection $[13,19]$. Interestingly, the detection of transmitted majority resistant variants was associated with a lower baseline plasma viral load and higher CD4 cell counts, suggesting a lower replicative capacity of transmitted resistant 
variants. Of note, this difference in the CD4 cell count was not determined by the clinician decision of when to start ART. The higher CD4 cell counts in the resistant group were conserved over six months on therapy, suggesting an immunological benefit in the short term. The detection of minority resistant variants by ASPCR was not associated to a worse virological response to ART in our study, contrary to the association found with the majority resistant variants. This finding could be explained by several hypotheses; the most commonly detected mutation by ASPCR was M184V. This mutation codes the resistance to 3TC and FTC but can reverse the resistance to AZT and tenofovir; Moreover, the decreased replicative capacity conferred by M184V is likely to result in lower viral loads and the continuation of 3TC-containining regimens despite the presence of $\mathrm{M} 184 \mathrm{~V}$ is not necessarily associated with a worse virological outcome [20]. It is also possible that the putative negative effect of the M184V on the virological response - if it exits- could have been masked by the antiviral effect of the other drugs, in majority protease inhibitors. Finally, we cannot exclude that the limited statistical power could have lead to the non-detection of the influence of minority variants in our study, since we could only perform ASPCR in a subgroup of patients. However, we maximised the statistical analyses by estimating slopes using repeated measurements of markers and taking into account undetectable viral load [14]. Furthermore, our data are very concordant with those recently reported from the Zurich-PHI Study [21] that found minority M184V variants in $14.9 \%$ of patients and minority K103N variants in $5.4 \%$ of patients without association to virological failure on early therapy. By contrast, Johnson et al [22] found an association between the detection of baseline minority resistant variants and virological response in patients enrolled in an abacavir/lamivudine/efavirenz trial. Hence, the heterogeneity due to the various treatment regimens used in our study population may explain the discrepant results with Johnston et al. by reducing the statistical power of our analysis. 
In conclusion, our study confirms the need for the detection of transmitted resistant variants by conventional sequencing methods in routine practice. The detection of minority resistant variants in nearly one-third of the investigated patients was not associated with the virological outcome on therapy but warrants further clinical validation in order to precise it's potential interest for the management of ART.

\section{Acknowledgements}

The authors thank all patients included in the study. We are indebted to Pascal Bonot and Marie-Hélène Shrive for excellent technical assistance. Authors’ contributions: O Peuchant developed and processed the allele-specific PCR; R Thiébaut developed the statistical model and participated in the writing of the manuscript; S Capdepont made the sequence analysis and phylogeny; V Lavignolle-Aurillac made the statistical analysis; D Neau and P Morlat were responsible for the clinical diagnosis and follow-up of the patients; F Dabis coordinated the Aquitaine Cohort; H Fleury directed the virology laboratory; and B Masquelier coordinated the study and wrote the manuscript.

\section{References}

1. Costagliola D, Descamps D, Assoumou L, et al. Prevalence of HIV-1 Drug Resistance in Treated Patients: A French Nationwide Study. J Acquir Immune Defic Syndr 2007.

2. Richman DD, Morton SC, Wrin T, et al. The prevalence of antiretroviral drug resistance in the United States. Aids 2004,18:1393-1401.

3. Masquelier B, Bhaskaran K, Pillay D, et al. Prevalence of transmitted HIV-1 drug resistance and the role of resistance algorithms: data from seroconverters in the CASCADE collaboration from 1987 to 2003. J Acquir Immune Defic Syndr 2005,40:505-511.

4. Wensing AM, van de Vijver DA, Angarano G, et al. Prevalence of drug-resistant HIV-1 variants in untreated individuals in Europe: implications for clinical management. J Infect Dis 2005,192:958-966.

5. Weinstock HS, Zaidi I, Heneine W, et al. The epidemiology of antiretroviral drug resistance among drug-naive HIV-1-infected persons in 10 US cities. $J$ Infect Dis 2004,189:2174-2180.

6. Brenner BG, Roger M, Routy JP, et al. High rates of forward transmission events after acute/early HIV-1 infection. J Infect Dis 2007,195:951-959. 
7. Chaix ML, Desquilbet L, Cottalorda J, et al. Sub-Optimal response to HAART in patients treated at time of primary HIV-1 infection and infrected with HIV resistant strains. Antivir Ther 2005,10:S127.

8. Little SJ, Holte S, Routy JP, et al. Antiretroviral-drug resistance among patients recently infected with HIV. $N$ Engl $J$ Med 2002,347:385-394.

9. Grant RM, Hecht FM, Warmerdam M, et al. Time trends in primary HIV-1 drug resistance among recently infected persons. Jama 2002,288:181-188.

10. Pillay D, Bhaskaran K, Jurriaans S, et al. The impact of transmitted drug resistance on the natural history of $\mathrm{HIV}$ infection and response to first-line therapy. Aids 2006,20:21-28.

11. Metzner KJ, Rauch P, Walter H, et al. Detection of minor populations of drugresistant HIV-1 in acute seroconverters. Aids 2005,19:1819-1825.

12. Lazaro E, Coureau G, Guedj J, et al. Change in T-lymphocyte count after initiation of highly active antiretroviral therapy in HIV-infected patients with history of Mycobacterium avium complex infection. Antivir Ther 2006,11:343-350.

13. Yeni P. Prise en charge thérapeutique des personnes infectées par le VIH. Paris: Médecine-Sciences Flammarion; 2006.

14. Metzner KJ, Bonhoeffer S, Fischer M, et al. Emergence of minor populations of human immunodeficiency virus type 1 carrying the M184V and L90M mutations in subjects undergoing structured treatment interruptions. J Infect Dis 2003,188:1433-1443.

15. Thiébaut R, Jacmin-Gadda H. Mixed models for longitudinal left-censored repeated measures. Computer Methods and Programs in Biomedicine 2004,74:255260.

16. Johnson J, Li J-F, Wei X, Lipscomb J, Smith A, Heneine W. Sensitive testing demonstrates a high prevalence of transmitted drug resistance among conventionally genotyped wildtype HIV-1 infections. Antivir Ther 2007,12:S46.

17. Coffin JM. HIV population dynamics in vivo: implications for genetic variation, pathogenesis, and therapy. Science 1995,267:483-489.

18. Wainberg MA. The impact of the M184V substitution on drug resistance and viral fitness. Expert Rev Anti Infect Ther 2004,2:147-151.

19. Hirsch MS, Brun-Vezinet F, Clotet B, et al. Antiretroviral drug resistance testing in adults infected with human immunodeficiency virus type 1: 2003 recommendations of an International AIDS Society-USA Panel. Clin Infect Dis 2003,37:113-128.

20. Castagna A, Danise A, Menzo S, et al. Lamivudine monotherapy in HIV-1-infected patients harbouring a lamivudine-resistant virus: a randomized pilot study (E184V study). Aids 2006,20:795-803.

21. Metzner KJ, Rauch P, Von Wyl V, et al. Prevalence of minority quasispecies of drug-resistant HIV-1 in patients with primary HIV-1 infection in Zurich in the years 2002-2006. Antivir Ther 2007,12:S47.

22. Johnson J, Li J-F, Wei X, et al. Low-frequency mutations substantially increase the prevalence of transmitted drug resistance and greatly strengthen the relationship between resistance mutations and virological failure. 14th conference on retroviruses and opportunistic infections. Abstr 639. Los Angeles, CA, USA 2007. 


\section{Legends to the figures}

Figure 1: Mean levels of plasma HIV RNA (A) and CD4 cell count (B) predicted by a piecewise linear model according to the presence of majority resistant variants. $\mathrm{N}=172$ patients, Aquitaine Cohort, 1996-2005.

Susceptible: baseline HIV-1 genotype predicting full sensitivity to the first-line ART. Resistance: baseline HIV-1 genotype predicting resistance to at least one drug of the first-line ART.

Figure 2: Mean levels of plasma HIV RNA (A) and CD4 cell count (B) predicted by a piecewise linear model according to the presence of minority resistant variants. $N=73$ patients, Aquitaine Cohort, 1996-2005.

Susceptible: baseline HIV-1 genotype predicting full sensitivity to the first-line ART.

Resistance: baseline HIV-1 genotype predicting resistance to at least one drug of the first-line ART. 
Table 1: Characteristics of the 172 patients included in the study

\begin{tabular}{|c|c|}
\hline & $\mathrm{N}(\%)$ \\
\hline \multicolumn{2}{|l|}{ Exposure category } \\
\hline Sex between men & $106(62 \%)$ \\
\hline $\begin{array}{l}\text { Sex between men } \\
\text { and women }\end{array}$ & $59(34 \%)$ \\
\hline Injecting drug use & $3(2 \%)$ \\
\hline Other/unknown & $4(2 \%)$ \\
\hline \multicolumn{2}{|l|}{ Sex } \\
\hline Male & $140(81 \%)$ \\
\hline Female & $32(19 \%)$ \\
\hline \multicolumn{2}{|l|}{ HIV-1 subtype } \\
\hline B & $142(83 \%)$ \\
\hline Non-B & $30(17 \%)$ \\
\hline \multicolumn{2}{|l|}{ Year of seroconversion } \\
\hline $\begin{array}{l}1996-1999 \\
2000-2003 \\
2004-2005\end{array}$ & $\begin{array}{l}77(45 \%) \\
72(42 \%) \\
23(13 \%)\end{array}$ \\
\hline Median Age (years, IQR) & $32(27-40.5)$ \\
\hline $\begin{array}{l}\text { Median CD4+ cell count } \\
(\text { cells } / \mu l)\end{array}$ & $426(314-579)$ \\
\hline Median plasma HIV-1 RNA & $4.7(4.1-5.3)$ \\
\hline \multicolumn{2}{|l|}{ First-line ART } \\
\hline 2-3 NRTIs & $49(28.5 \%)$ \\
\hline NRTIS+ NNRTI & $37(21.5 \%)$ \\
\hline NRTIS+PIs & $86(50 \%)$ \\
\hline $\begin{array}{l}\text { Resistance to at least one } \\
\text { drug of the regimen }\end{array}$ & $16(9.3 \%)$ \\
\hline
\end{tabular}

Abbreviations: ART: antiretroviral therapy; NRTI: nucleoside reverse transcriptase inhibitor; NNRTI: non-nucleoside reverse transcriptase inhibitor; PI: protease inhibitor 
Figure 1 A

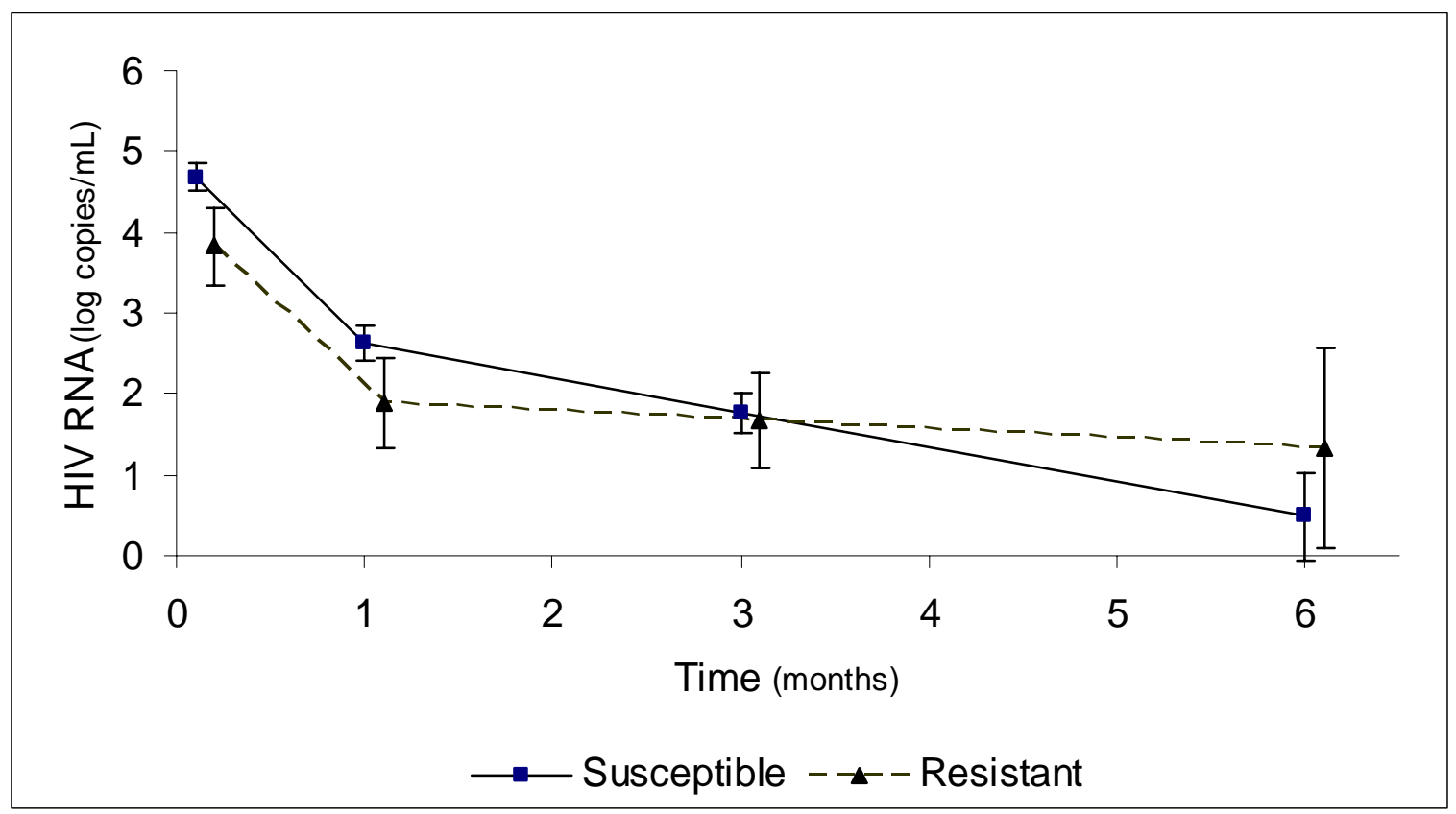

Figure 1 B

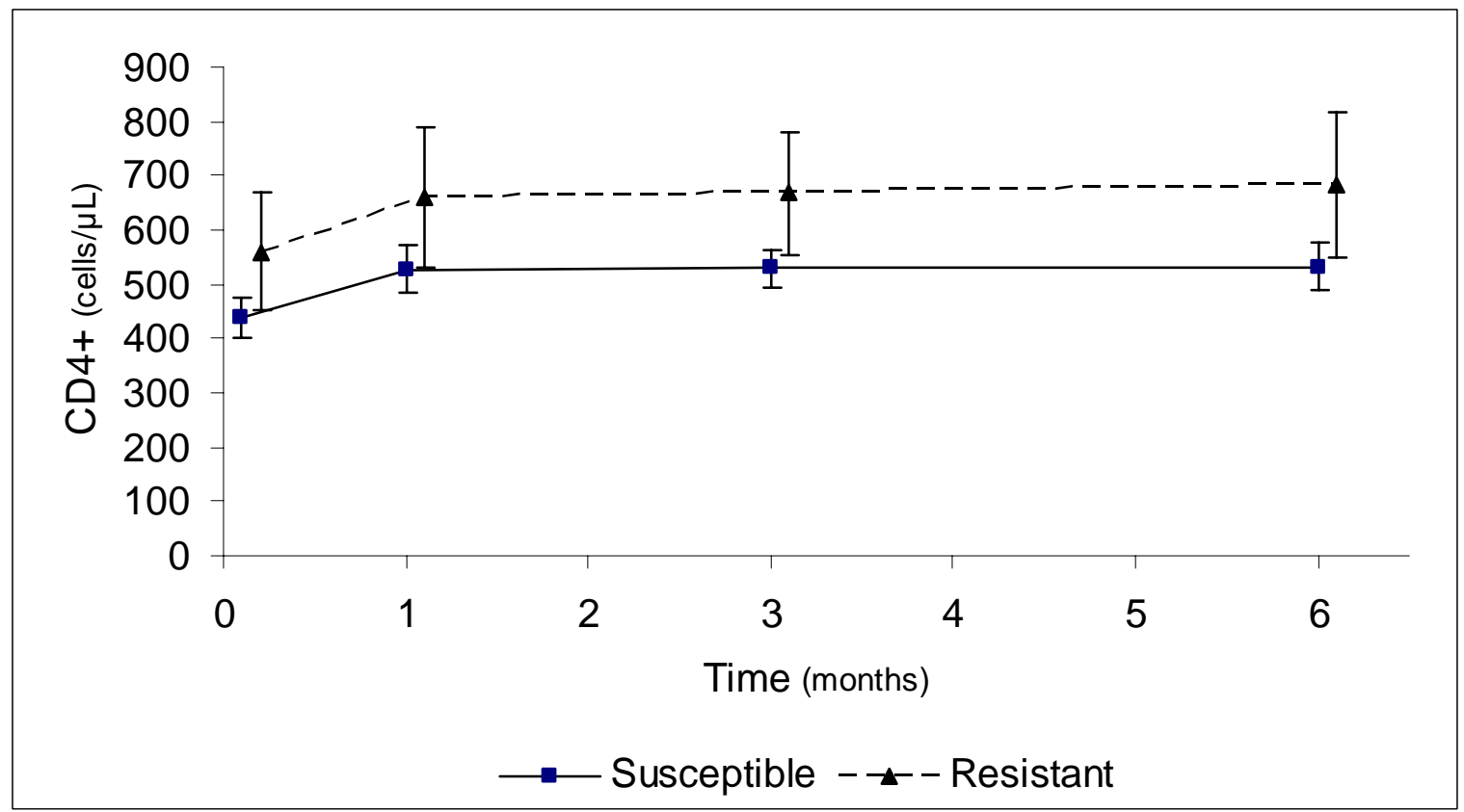


Figure 2A

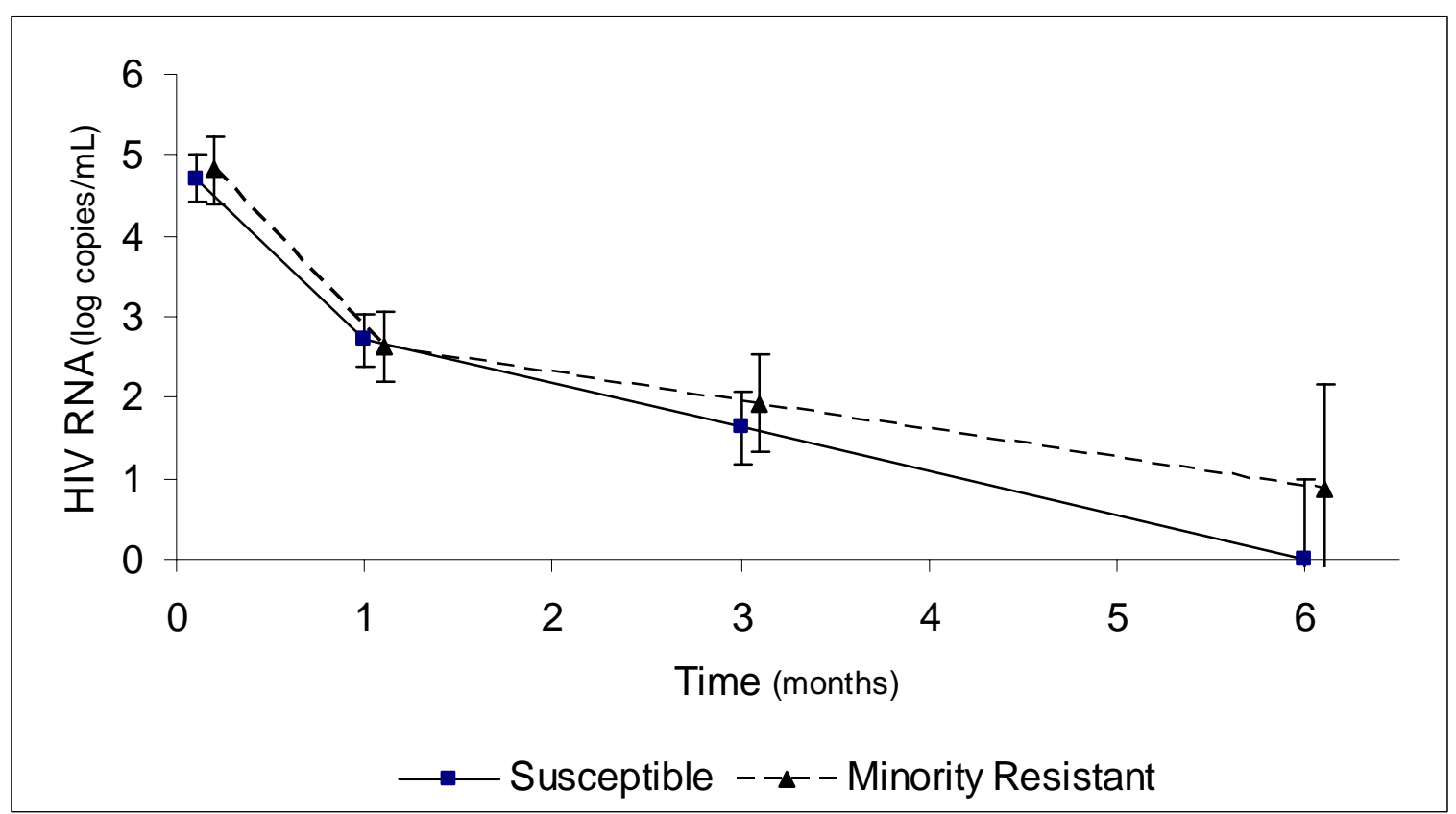

Figure 2B

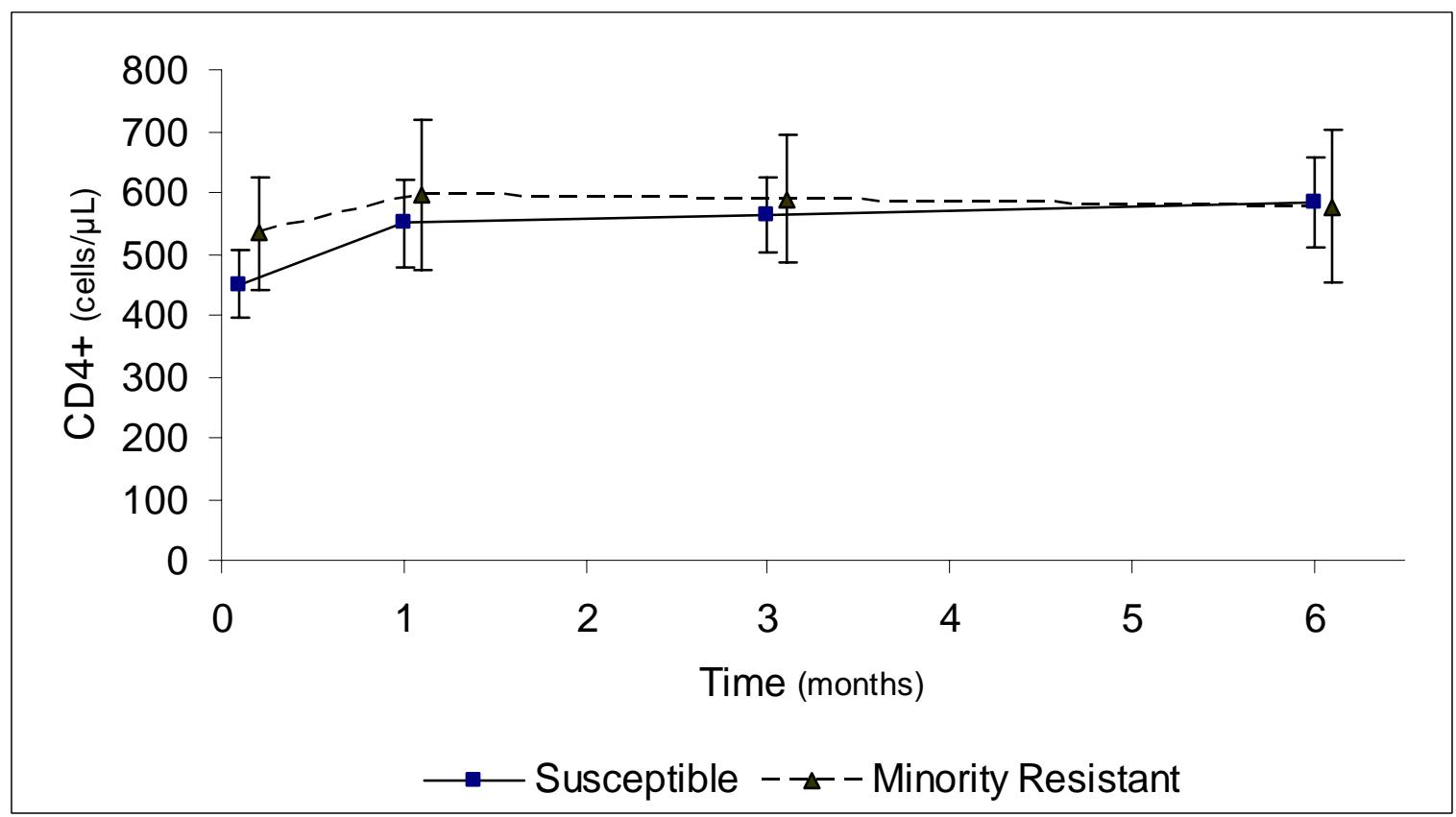




\section{Appendix}

Composition of the GECSA (Groupe d’Epidémiologie Clinique du SIDA en Aquitaine) ANRS CO3 Aquitaine cohort:

Coordinator: F. Dabis. Epidemiology and Methodology: G. Chêne, F. Dabis, S. LawsonAyayi, R. Thiébaut, M. Winnock. Infectious diseases and Internal medicine: M. Bonarek, F. Bonnal, F. Bonnet, N. Bernard, O. Caubet, L. Caunègre, C. Cazanave, J. Ceccaldi, P. Couzigou, FA Dauchy, C. De La Taille, S. De Witte, M. Dupon, P. Duffau, H. Dutronc, S. Farbos, MC. Gemain, C. Greib, K. Lacombe, D. Lacoste, S. Lafarie-Castet, P. Loste, D. Malvy, P. Mercié, P. Morlat, D. Neau, A. Ochoa, JL. Pellegrin, JM. Ragnaud, S. Tchamgoué, J.F. Viallard. Immunology: P. Blanco, JF. Moreau. Virology: H. Fleury, ME. Lafon, B. Masquelier, I. Pellegrin. Pharmacology: D. Breilh. Pharmacovigilance: G. MiremontSalamé. Data collection and Data management: MJ. Blaizeau, M. Decoin, S. Delveaux, S. Geffard, S. Gillet, C. Hannapier, S. Labarrère, V. Lavignolle-Aurillac, B. UwamaliyaNziyumvira. Data processing: G. Palmer, D. Touchard. 